\title{
Editorial
}

\section{A HOT opportunity}

The Java phenomenon means that programmers that once laughed at garbage collection and strong typing have started to use it daily, and this opens up a wonderful opportunity for the functional programming community.

Bob Harper coined the acronym HOT to summarise much of what functional programmers have to offer the world: expertise in languages that are Higher-Order and Typed. Bob argued for a broad interpretation of these terms, so that HigherOrder includes languages where objects contain methods (even though functions are not first-class citizens), and Typed includes both static and dynamic typing. By these criteria Java is HOT, and so are Haskell, ML and Scheme.

Not all functional languages of interest are all that HOT. Ericsson is using the functional and concurrent language Erlang to build phone switches containing hundreds of thousands of lines of code. Erlang is a first-order language with dynamic types (though recent work has added higher-order, and static types are in the offing).

The key to Erlang's success is similar to Java's: both have thrived by focussing on an application area, the web for Java, telephony for Erlang. Those who strive to eke another $5 \%$ speed-up out of their compilers should note another similarity between Java and Erlang: both achieved their initial success based on byte code interpreters of modest efficiency (though fast native code compilers for both are coming along). We should continue to seek 'killer apps' for functional programming.

The Journal of Functional Programming encourages all attempts at outreach, from our area to others, from theory to practice. JFP has published papers on the higher-order and typed aspects of Modula-3, Algol and Actors. We have run (or are about to run) papers on applications to animation, artificial intelligence, music, protein analysis, scientific programming and text processing. We have had a special issue devoted to type systems for object-oriented programming, and another to applications.

As always, we remain a broad church. Send us your HOT papers, your application papers, your papers on links to other areas. Send us papers about languages typed or untyped, strict or lazy, pure or stateful, sequential or concurrent.

\section{The journal}

On the home front, there are some changes to announce. Sad to say, Henk Barendregt, due to pressures of work, has resigned as an editor of JFP. However, we are delighted that he has agreed to remain as editor of our Theoretical Pearls column. 
So continue to send Henk any beautiful and brief candidates for his column, but please send other submissions to one of the other JFP editors. Please join us in thanking Henk for his help in founding JFP, and for his seven years of devoted service.

And please join us in welcoming our new editor, Thierry Coquand. Thierry brings special expertise in the HOT area of type theory, and is generally knowledgeable in all things theoretical. To give Thierry the warmest welcome, send him a paper today; submission details are on the inside back cover, addresses on the inside front. The theory editor is dead, long live the Thierry editor!

Please also join us in welcoming Simon Thompson as book review editor. Publishers are keen to give away books in return for reviews, we are keen to run discriminating reviews, and the reviewer gets to keep the book. Let Simon know if you'd like to register as a reviewer, if there's some book you want to review, or if there's a book you'd like to see us review. His address is inside the front cover.

This is also a good point to thank Richard Bird for his continuing effort as editor of Functional Pearls. If you have a contribution that is small, rounded, and pleasant, you'll also find his address, too, on the inside front. Richard continues to contribute regular columns, setting a high standard for the rest of us.

And when you do send a paper, just how long will it take to get a response? Answer: About three months, on average. We at JFP have no patience for journals that sit on papers for years. The editors all aim to reach a decision (accept, revise or reject) on each paper within four months of receipt, a goal which Simon, Paul and John met for every paper processed in the last year, and which Phil failed to meet just once (by four days). To achieve this, we ask our reviewers to acknowledge papers immediately and to provide reviews promptly, typically within a month. And we get back to them if we don't hear from them.

This leads us to our penultimate thank you: to the reviewers who make the process work. Without them, our science would grind to a halt.

A venerable curse reads "May you live in interesting times". The written word is increasingly viewed on the screen rather than on the page, and our web site at www.dcs.glasgow.ac.uk/jfp is increasingly a part of what we do. Nonetheless, we intend to publish as atoms rather than as bits for a while longer.

Atoms or bits, journals will remain at the core of scientific progress. Their key ingredients are not ink and pulp, nor Latex and HTML, but writers, reviewers and readers. Our final thank you is to you, for your contribution to JFP. We look forward to taking, with you, our next step into interesting times.

Philip Wadler for the editors of JFP 